\title{
Budding and vesiculation induced by conical membrane inclusions
}

\author{
Thorsten Auth and Gerhard Gompper \\ Institut für Festkörperforschung and Institute for Advanced Simulations, Forschungszentrum Jülich, D-52425 Jülich, Germany
}

(Received 22 January 2009; published 3 September 2009)

\begin{abstract}
Conical inclusions in a lipid bilayer generate an overall spontaneous curvature of the membrane that depends on concentration and geometry of the inclusions. Examples are integral and attached membrane proteins, viruses, and lipid domains. We propose an analytical model to study budding and vesiculation of the lipid bilayer membrane, which is based on the membrane bending energy and the translational entropy of the inclusions. If the inclusions are placed on a membrane with similar curvature radius, their repulsive membranemediated interaction is screened. Therefore, for high inclusion density the inclusions aggregate, induce bud formation, and finally vesiculation. Already with the bending energy alone our model allows the prediction of bud radii. However, in case the inclusions induce a single large vesicle to split into two smaller vesicles, bending energy alone predicts that the smaller vesicles have different sizes whereas the translational entropy favors the formation of equal-sized vesicles. Our results agree well with those of recent computer simulations.
\end{abstract}

DOI: 10.1103/PhysRevE.80.031901

PACS number(s): 87.16.D-, 87.17.-d, 82.70.Uv

\section{INTRODUCTION}

Cell membranes contain large amounts of proteins within or attached to the lipid bilayer [1]. The distribution of the proteins is not necessarily homogeneous, which can have important functional consequences. For example, proteins with an intrinsic curvature couple to the bilayer conformation $[2-8]$; on the one hand, such proteins are preferably found on similarly curved parts of the membrane [9], on the other hand, the proteins deform the membrane locally $[10,11]$. Asymmetric, curved proteins can regulate the polymerization of the three-dimensional cytoskeleton of the cell [12] and control intracellular transport via endocytosis $[13,14]$. Virus endocytosis can occur via the same mechanism $[15,16]$. The conical inclusions in our model mimic asymmetric proteins within the bilayer [7], proteins or polymers attached to the bilayer $[4,17,18]$, curved lipid domains [19-22], and viruses that bind to the membrane [15].

The interaction between the inclusions in a lipid bilayer is mediated by membrane deformations and thermal undulations $[23,24]$, in addition to surface tension [2] and possible direct interactions that we do not consider in this paper. The deformation-induced, pairwise interaction of curved inclusions occurs in the absence of thermal membrane undulations and is usually repulsive $[25,26]$; in a planar membrane it is long range [25-27]. However, the interactions can be strongly screened if the average curvature of the membrane and the protein curvature are similar [28-30]. One obvious example for strongly screened interactions are inclusions that are placed on a vesicle with similar curvature radius [30]. Screening can also be achieved by many-body interaction in clusters of inclusions [29,31]. At finite temperature, Casimirtype interactions due to membrane undulations generate attraction [25,26,32-35].

Curvature generation by inclusions and induced budding in lipid bilayer membranes has been reported in many experimental studies of biological and biomimetic systems $[7,11,13,14,18]$. Computer simulations allow to study the membrane-mediated interaction between the inclusions in detail without the presence of direct interactions. Recently, bud formation by curved inclusions has been investigated with computer simulations [36,37]. It was found that the inclusions on the buds have a higher density than they had in the initially nearly flat membrane [36]. This might appear to be a result of undulation-induced attraction that in consequence leads to clustering of the inclusions and to budding.

Such systems and processes can be studied theoretically on the basis of an elastic membrane that is characterized by its bending rigidity, $\kappa$, and Gaussian saddle-splay modulus, $\bar{\kappa}$, with curved inclusions that consist of sections of a sphere with a given opening angle. We demonstrate that bud formation can already be well understood on the basis of the membrane deformation alone. We show that the higher inclusion density on the bud is a result of a screened repulsive interaction. We further argue that the budding pathway plays an important role for the bud size. This allows us to predict a range of possible bud radii for a given system, which nicely agrees with recent simulation results [36].

At finite temperature, the inclusions can exist in a fluid and in a crystalline phase, which depends on the strength of their repulsive interaction. We construct an approximate freeenergy functional that takes into account for the bending energy as well as the translational entropy of the inclusions. We calculate a phase diagram for the fission of a single vesicle of given size and for given number and geometry of the inclusions. The inclusion entropy plays a decisive role for the sizes of the smaller vesicles into which a larger vesicle may split.

\section{MEMBRANE BENDING ENERGY}

\section{A. Membrane shape near inclusions in a lipid bilayer}

The bending energy $\mathcal{E}$ of a lipid bilayer is given by the integral over the entire membrane area,

$$
\mathcal{E}=\int d S\left(2 \kappa H^{2}+\bar{\kappa} K\right),
$$

where $\kappa$ is the bending rigidity, $\bar{\kappa}$ is the saddle-splay modulus, $H=\left(c_{1}+c_{2}\right) / 2$ is the mean curvature, $K=c_{1} c_{2}$ is the 

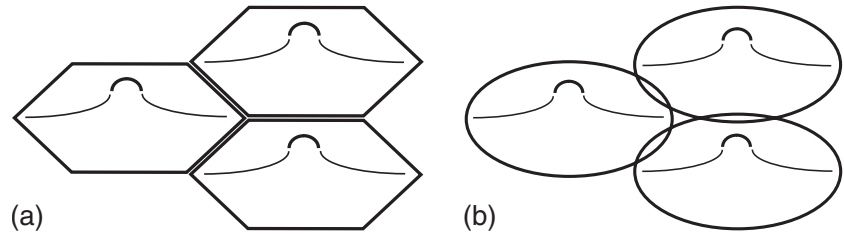

FIG. 1. (a) Membrane deformations induced by curved inclusions in a planar membrane. The inclusions have a repulsive interaction potential that decreases with the distance between the inclusions, $d$, like $V \sim d^{-2}$. To minimize the bending energy, the inclusions order in a hexagonal structure. (b) The hexagons are approximated by overlapping circles that have the same projected area.

Gaussian curvature, and $c_{1}$ and $c_{2}$ are the principal curvatures at each point of the membrane. The integral over the Gaussian curvature is determined by the topology of the membrane and by the geodesic curvature at the boundary. In our case, the geodesic curvature is given by the geometry of the inclusions, so that in general this term of the integral over the membrane shape does not need to be calculated explicitly. For bud formation, we neglect the constant contribution of the Gaussian saddle-splay modulus.

In order to minimize the bending energy, the inclusions preferably order on a hexagonal lattice [Fig. 1(a)]; therefore it is a natural assumption that the symmetry axis is oriented normal to the local tangent plane of the vesicle on which the inclusions are placed. To calculate the deformation energy, we approximate the hexagons with overlapping circles that have the same projected area [Fig. 1(b)].

If there are no overhangs, the membrane conformation can be described in Monge parametrization by a height field, $h(x, y)$, over a planar reference surface. For an almost planar membrane, the bending energy of the membrane is

$$
\mathcal{E}=\frac{1}{2} \kappa \int d A[\Delta h(\boldsymbol{\rho})]^{2}, \quad[\boldsymbol{\rho}=(x, y)],
$$

with $\int d A$ the integral over the reference plane. Minimization of the bending energy gives the biharmonic Euler-Lagrange equation

$$
\Delta^{2} h(\boldsymbol{\rho})=0 .
$$

In cylindrical coordinates, the general solution of Eq. (3) is

$$
h(\rho)=\frac{1}{4} \rho^{2}\left(2 C_{2}-C_{3}\right)+C_{4}+\left(C_{1}+\frac{1}{2} \rho^{2} C_{3}\right) \ln (\rho)
$$

with the four integration constants $C_{1}$ to $C_{4}$ [38].

The boundary conditions that are imposed on the membrane are sketched in Fig. 2. The radius of the inner boundary, $\rho_{i}=r_{i} \sin (\alpha)$, and the slope of the membrane at the inner boundary, $h^{\prime}\left(\rho_{i}\right) \equiv a=-\tan (\alpha)$, are determined by the inclusion geometry. For $n \approx 4 \pi R^{2} \sigma$ inclusions on a vesicle with radius $R$ and surface number density $\sigma$ of the inclusions, the radius of the outer boundary is $\rho_{o} \approx R \sin (\beta)$ with $\beta=\arccos [(n-2) / n]$; the slope of the membrane at the outer boundary is $h^{\prime}\left(\rho_{o}\right) \equiv b=-\tan (\beta)$. For inclusions on a planar membrane, the latter expressions simplify to $\rho_{o} \approx 1 /(\pi \sigma)^{(1 / 2)}$ and $b=0$. The remaining two boundary con-

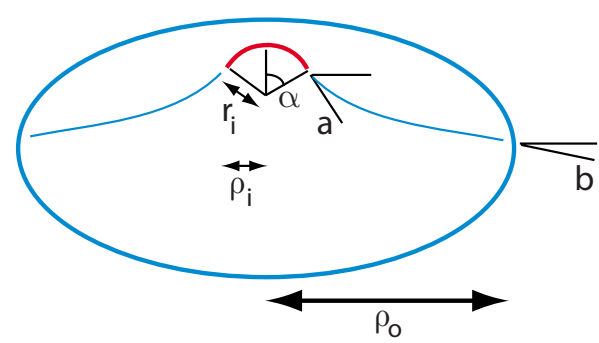

FIG. 2. (Color online) Curved inclusion and resulting membrane deformation. The inclusion geometry is characterized by the curvature radius, $r_{i}$, the opening angle, $\alpha$, and the projected inclusion radius, $\rho_{i}=r_{i} \sin (\alpha)$. The size of the corresponding membrane patch is $\rho_{o}$, the slope of the membrane at the inclusion is $a=-\tan (\alpha)$, and the slope of the membrane at the outer boundary is $b(b=0$ for inclusions on a planar membrane).

ditions are given by fixing the membrane height at the inner (or equivalently at the outer) boundary and minimizing the energy with respect to the height of the inclusion above the vesicle (i.e., the height difference between both boundaries), which implies $h\left(\rho_{i}\right)=0$ at the inclusion and $\left.\partial_{\rho} \Delta h(\rho)\right|_{\rho_{o}}=0$ at the outer boundary.

Equation (4) together with the boundary conditions gives the shape of the deformation,

$$
h(\rho)=\frac{\left(\rho^{2}-\rho_{i}^{2}\right)\left(b \rho_{o}-a \rho_{i}\right)+2 \rho_{o} \rho_{i}\left(a \rho_{o}-b \rho_{i}\right) \ln \left(\rho / \rho_{i}\right)}{2\left(\rho_{o}^{2}-\rho_{i}^{2}\right)},
$$

and the corresponding bending-energy cost,

$$
\mathcal{E}\left(\rho_{o}, b\right)=\frac{\kappa}{2} \int_{\rho_{i}}^{\rho_{o}} d \rho\left[\Delta_{r} h(\rho)\right]^{2}=\frac{2 \pi \kappa\left(b \rho_{o}-a \rho_{i}\right)^{2}}{\left(\rho_{o}^{2}-\rho_{i}^{2}\right)} .
$$

The energy is a function of $\rho_{o}$ and $b$, which depend on the inclusion density, while all other quantities are intrinsic properties of membrane and inclusions. For a single inclusion in an infinite planar membrane, $b=0$ and $\rho_{o} \rightarrow \infty$, the bending energy vanishes and the membrane deformation is catenoidlike, $h(\rho)=a \rho_{i} \ln \left(\rho / \rho_{i}\right)$. Note that in a pairwise approximation, the interaction energy for two inclusions in a planar membrane $(b=0)$ decays like $d^{-2}$ for large distances between the inclusions (large $\rho_{o}=d / 2$ ).

\section{B. Optimal, low, and high inclusion density}

For inclusions on a vesicle, the membrane shape and the minimal bending energy (assuming that the inclusions have maximal mutual distances) can be calculated using Eqs. (5) and (6). For $b \rho_{o}=a \rho_{i}$, the membrane around the inclusion has almost catenoid shape [39]; the catenoid is a minimal surface without bending-energy cost. If the entire vesicle is covered with inclusions and catenoids such that the bending energy is zero [Fig. 3(b)], the inclusions have optimal density.

For lower inclusion densities, in a first approximation the catenoid shape borders on a spherical shape with the curvature radius of the vesicle. The bending energy of a vesicle that is decorated with curved inclusions is reduced by the 

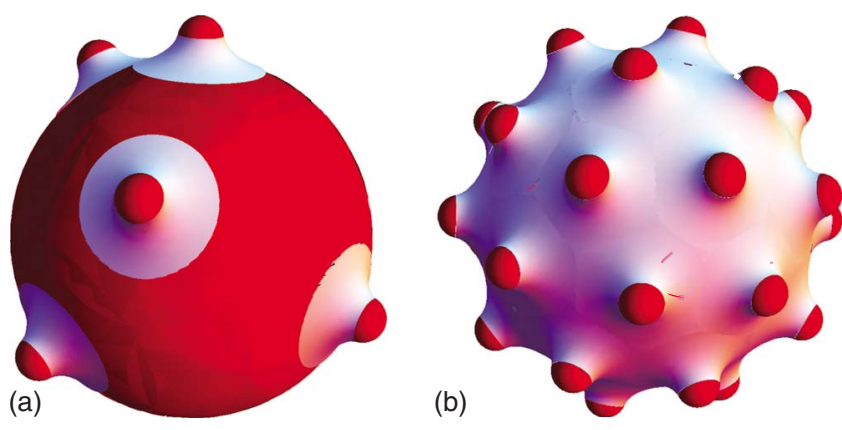

FIG. 3. (Color online) (a) Vesicle decorated with curved inclusions. Around each inclusion, the membrane can be modeled by segments of the catenoid minimal surface (white). The total bending energy is $\mathcal{E}=8 \pi \kappa\left(1-S_{\mathrm{cat}} / S_{\mathrm{sph}}\right)$, where $S_{\mathrm{cat}} / S_{\mathrm{sph}}$ is the area fraction of the vesicle that is covered with inclusions and catenoidal patches. (b) Vesicle decorated with inclusions at optimal density; the bending energy of the lipid bilayer membrane vanishes.

fraction of the sphere's surface area that is covered by the inclusions and the catenoid-shaped membrane segments; see Fig. 3. Therefore, for low inclusion density, the bending energy of the decorated spherical vesicle is in the range $0<\mathcal{E}<8 \pi \kappa$. In the full solution, which is given by Eq. (5) and will be used in the remainder of the paper, there is no jump in the mean curvature from $H=0$ to $H=1 / R$ between the catenoid and a sphere as sketched in Fig. 3 but rather a smooth transition from zero to finite mean curvature.

For inclusion densities that are higher than the optimal density, due to the boundary conditions no solution can be constructed by matching of catenoids. In this case, the bending energy always has a finite value that can exceed the bending energy of a bare vesicle.

The minimal bending energy of a vesicle with inclusions is shown in Fig. 4 as function of the number of inclusions, $n$, and the vesicle radius, $R$. We find degenerate zero-energy

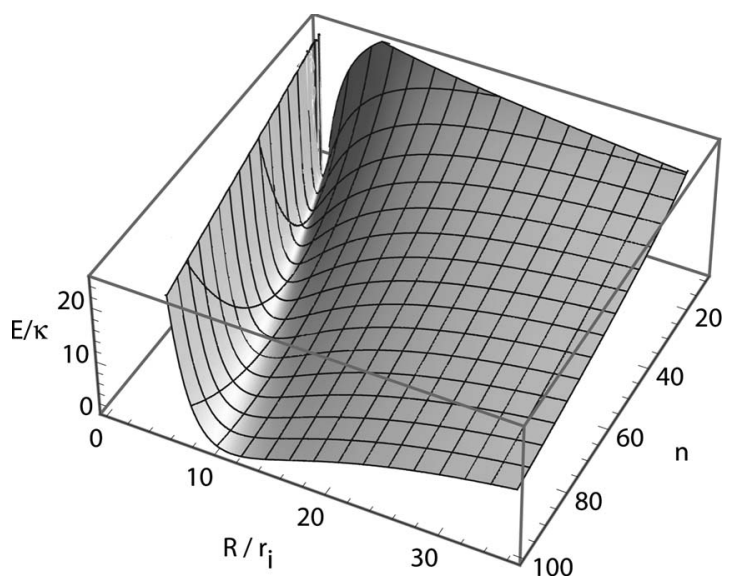

FIG. 4. Normalized bending energy, $E / \kappa$, of a vesicle with radius $R$ with $n$ inclusions ( $r_{i} \approx 5.5 \mathrm{~nm}, \alpha=0.64$ ). There is a region of low inclusion density at large $R$ with $0<\mathcal{E}<8 \pi \kappa$, which is delineated by a line of zero-energy ground states from a region of high inclusion density at small $R$, where also bending energies $\mathcal{E}>8 \pi \kappa$ can be found. (The high energies that are cut off at small $n$ and large $R$ mark the breakdown of the small-curvature expansion of the bending energy.) ground states that have optimal inclusion density with an approximately linear dependence $R(n)$, where [40]

$$
\begin{aligned}
& R \approx|a| \rho_{i} n / 4 \approx 1 /\left(\pi \sigma|a| \rho_{i}\right)=(\cos \alpha) /\left(\pi \sigma \sin ^{2} \alpha\right)\left(1 / r_{i}\right) \\
& \text { and } n \approx 4 /\left(\pi \sigma a^{2} \rho_{i}^{2}\right)=\left(4 \cos ^{2} \alpha\right) /\left(\pi \sigma \sin ^{4} \alpha\right)\left(1 / r_{i}^{2}\right)
\end{aligned}
$$

The natural spontaneous curvature of the bilayer for given inclusion density and geometry is $c_{0}=1 / R_{0} \approx \pi \sigma|a| \rho_{i}$.

The same value for the inclusion density can be high, optimal, or low, depending on the radius of the vesicle on which the inclusions are placed. The smaller the radius of the vesicle, the larger the value of the optimal density. In a planar membrane, the slopes of two adjacent catenoidlike deformations cannot be matched for any finite distance between the inclusions. Therefore, the inclusions are always in the high-density regime in this case.

\section{Budding and vesiculation}

Bud formation does not occur for a vesicle with low inclusion density and bending energy, $0<\mathcal{E}<8 \pi \kappa$, because this would lead to an increase in the total bending energy [41]. However, for high inclusion density $\left[n \gtrsim 4 R /\left(|a| \rho_{i}\right)\right.$; see Eq. (7)], the system can always reach a state of lower bending energy if small vesicles bud from the main vesicle. The set of smaller vesicles into which a large vesicle with high inclusion density splits up is not uniquely determined from bending energy alone, because the states of vanishing bending energy are degenerate. A natural assumption is that the vesicle will split into one large "mother" vesicle and one or more small "daughter" vesicle(s) of equal size, such that the total bending energy vanishes and the membrane area is kept constant. In Fig. 5, we show the radii of the mother and daughter vesicles as function of the number of inclusions. For a given number of $n_{v}-1$ daughter vesicles, there is a maximal number of inclusions $n_{\max }=n_{v}^{1 / 2} w R\left[w=4 /\left(|a| \rho_{i}\right)\right]$ that still allows to obtain a zero-energy state, for which mother and daughter vesicles have equal sizes.

If the system can split up into $n_{v}$ smaller vesicles, it can also split up into a larger number of small vesicles [42]. For a vesicle with total number of inclusions $n=n_{+}+\left(n_{v}-1\right) n_{-}$ and radius $R=\left[R_{+}^{2}+\left(n_{v}-1\right) R_{-}^{2}\right]^{1 / 2}$, bending energy minimization predicts for the radii and inclusion number on mother $\left(R_{+}, n_{+}\right)$and daughter $\left(R_{-}, n_{-}\right)$vesicles:

$$
\begin{aligned}
& n_{+}=\frac{n+\left(n_{v}-1\right)^{1 / 2}\left(n_{v} w^{2} R^{2}-n^{2}\right)^{1 / 2}}{n_{v}}, \\
& n_{-}=\frac{n-\left(n_{v}-1\right)^{-1 / 2}\left(n_{v} w^{2} R^{2}-n^{2}\right)^{1 / 2}}{n_{v}}, \\
& R_{+}=\frac{n+\left(n_{v}-1\right)^{1 / 2}\left(n_{v} w^{2} R^{2}-n^{2}\right)^{1 / 2}}{n_{v} w}, \\
& R_{-}=\frac{n-\left(n_{v}-1\right)^{-1 / 2}\left(n_{v} w^{2} R^{2}-n^{2}\right)^{1 / 2}}{n_{v} w} .
\end{aligned}
$$

Because of the degeneracy of the states with vanishing bending energy, thermal fluctuations and the budding pathway 


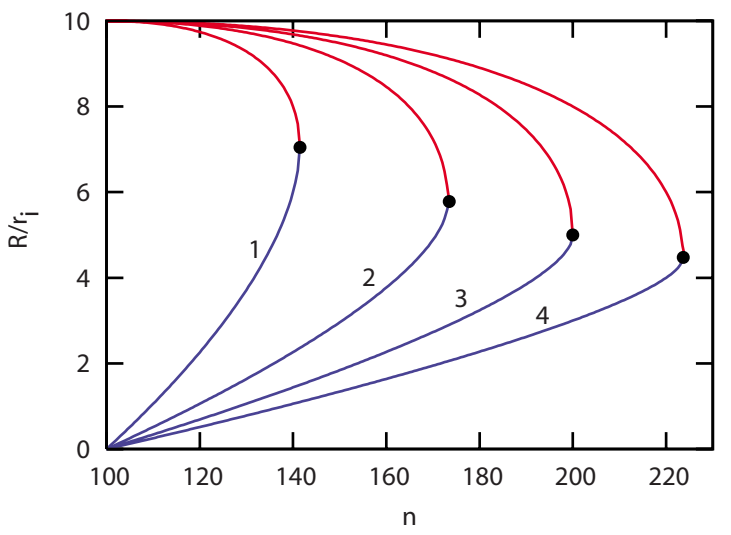

FIG. 5. (Color online) A single vesicle of radius $R=10 r_{i}$ with $n$ inclusions splits into one large "mother" vesicle and several small "daughter" vesicles; in the figure the cases of one, two, three, and four daughter vesicles are shown. The same parameters as in Fig. 4 are used. For 100 inclusions, the initial vesicle has vanishing bending energy. For more than 100 inclusions, the sizes of mother and daughter vesicles are plotted. The upper branch always gives the radius of the mother vesicle; the lower branch is the size of the daughter vesicles. For a fixed number $n_{v}-1$ of daughter vesicles (as indicated), there is a maximum number of inclusions that allows the formation of a state with vanishing bending energy (for which mother and daughter vesicles have equal sizes; filled circles).

play a decisive role to determine how a large vesicle with high inclusion density splits up into smaller vesicles. Note that the results of our analytical model so far do not depend on the value of the bending rigidity of the bilayer.

\section{Inclusion clusters}

A direct attractive interaction between inclusions can induce cluster formation [43]. In this case, the preferred curvature radius, $R_{0}$, not only depends on the number and geometry of the inclusions, but also on the cluster size. For given inclusion curvature radius $r_{i}$, opening angle $\alpha_{0}$, and fixed density $\sigma_{0}$, inclusion clusters with a larger opening angle $\alpha$ and reduced density, $\sigma=\left(1-\cos \alpha_{0}\right) /(1-\cos \alpha) \sigma_{0}$, have a stronger effect on the curvature of the membrane than homogeneously distributed inclusions. The preferred curvature radius for clusters with opening angle $\alpha$ is

$$
\begin{aligned}
R(\alpha) & =[(1-\cos \alpha) \cos \alpha] /\left[\left(1-\cos \alpha_{0}\right) \sin ^{2} \alpha\right] /\left(\pi r_{i} \sigma_{0}\right) \\
& \equiv f(\alpha) /\left(\pi r_{i} \sigma_{0}\right) .
\end{aligned}
$$

We call the normalized curvature radius $f(\alpha)$ the coagulation factor, because it multiplies the curvature radius for the reference inclusion density and opening angle $\alpha_{0}[44,45]$; see Fig. 6.

For a fixed number $n_{0}$ of inclusions, the preferred curvature radius decreases when aggregates are formed; i.e., the efficiency with which the inclusions influence the membrane curvature increases. Cluster formation, therefore, also shifts the high-density regime to smaller numbers of inclusions for the same vesicle radius, $n_{0} \gtrsim f(\alpha) 4 R / r_{i}$, and may cause a large vesicle to break up into smaller vesicles.

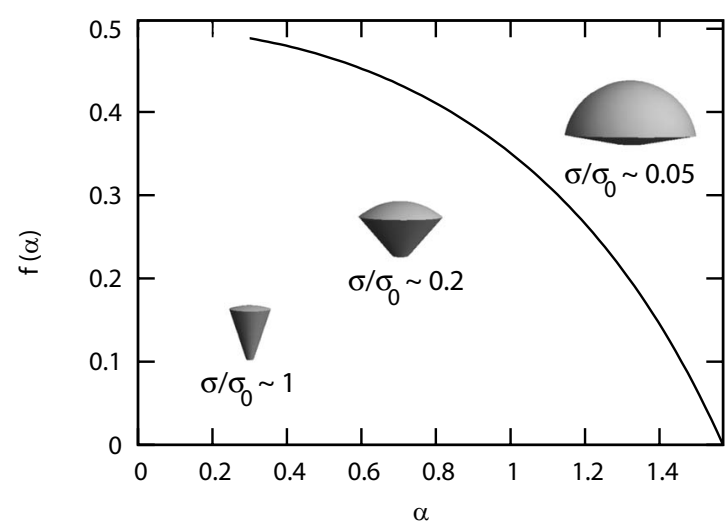

FIG. 6. The coagulation factor, $f(\alpha)=R(\alpha) \pi r_{i} \sigma_{0}$, describes the dependence of the optimal vesicle radius, $R_{0}$, on the degree of aggregation of the inclusions in the membrane. The total inclusion area is kept constant and the reference density $\sigma_{0}$ corresponds to $\alpha_{0}=0.1 \pi$.

\section{THERMAL FLUCTUATIONS}

For finite temperature, the translational entropy of the inclusions contributes to the free energy. We distinguish a crystalline hexagonal phase and a disordered fluid phase for which we construct free-energy functionals. Phase diagrams have been calculated more rigorously in Ref. [46] in the limiting case of an almost planar membrane and for inclusions that are slightly stiffer than the membrane and weakly curved-but not in the context of budding.

We neglect the interaction between inclusions by thermal membrane undulations. For a pair of inclusions and an arbitrary orientation of the inclusion axis, to lowest order of $\rho_{i}^{2} / d^{2}$ the deformation energy is $[25-27,47]$

$$
\mathcal{E}_{\mathrm{def}}=8 \pi \kappa \alpha^{2} \frac{\rho_{i}^{4}}{d^{4}}
$$

and the undulation-induced interaction energy $[25,34,35,48]$

$$
\mathcal{F}_{\text {und }}=-6 k_{B} T \frac{\rho_{i}^{4}}{d^{4}} .
$$

The ratio of the membrane deformation-induced repulsion to the undulation-induced attraction in a planar membrane is $4 \pi \kappa \alpha^{2} /\left(3 k_{B} T\right)$. The undulation-induced attraction can be neglected if it is one order of magnitude smaller than the deformation interaction; for $\kappa=10 k_{B} T$ this is the case for $\alpha \gtrsim 0.5$, for $\kappa=20 k_{B} T$ already for $\alpha \gtrsim 0.35$. For low inclusion densities, the undulation-induced interaction energy decays with the square of the density while the free energy due to the inclusion entropy is of the order of $k_{B} T$; in case of the phase diagrams in Fig. 11, the undulation free energy is about $10^{-4} k_{B} T$.

\section{A. Inclusion effective pair potential and effective hard-disk radius}

For inclusions on a hexagonal lattice, each inclusion corresponds to three pair interactions and a radius of the deformation patch, $\rho_{o}$, that is half the distance between the inclu- 


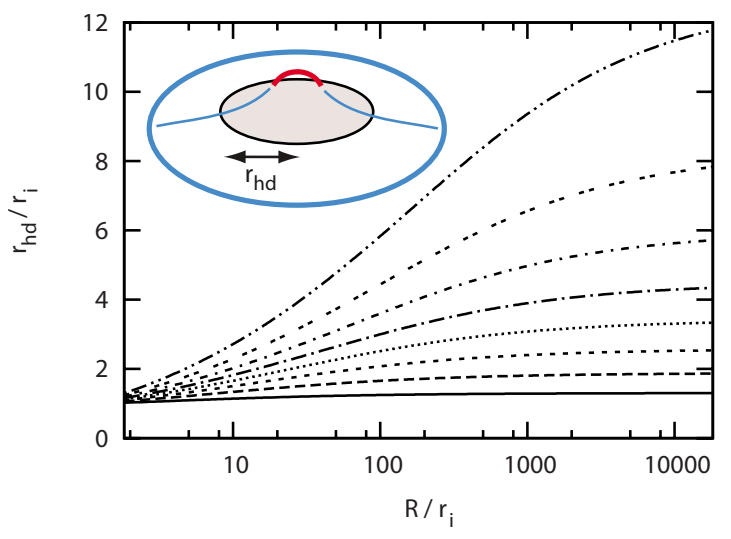

FIG. 7. (Color online) Effective hard-disk radii for inclusions on a vesicle as function of the vesicle's radius, $R$ [see Eq. (12)]. All inclusions have the curvature radius, $r_{i}=5.5 \mathrm{~nm}$, and the opening angles $\alpha=0.4 \pi, \alpha=0.35 \pi, \ldots, \alpha=0.05 \pi$ (from top to bottom). The projected inclusion radii are in the range $0.86 \mathrm{~nm}<\rho_{i}<5.2 \mathrm{~nm}$; the membrane bending rigidity is $\kappa=12 k_{B} T$.

sions. The effective pair potential, obtained from Eq. (6), is thus

$$
\begin{array}{cc}
u(d)=\frac{2 \pi \kappa\left(b d-2 a \rho_{i}\right)^{2}}{3\left(d^{2}-4 \rho_{i}^{2}\right)} & \text { if } b d \leq 2 a \rho_{i} \\
u(d) \approx 0 & \text { if } b d>2 a \rho_{i} .
\end{array}
$$

For inclusions on a planar membrane $(b=0)$ and for large $d$, i.e., for $d \gg \rho_{i}$, the repulsive interaction potential decays with a power law, $u \sim d^{-2}$.

To determine the free energy of this system, we employ the method developed for suspensions of repulsive colloids [49]; i.e., we mimic the interaction potential by hard disks with an effective radius, $r_{\mathrm{hd}}$. The radius is calculated from a comparison of the membrane deformation energy with the thermal energy, $k_{B} T$. We use a modified Barker-Henderson method that is appropriate for soft potentials [45,49],

$$
r_{\mathrm{hd}}=\frac{1}{2} \int_{0}^{\rho_{u}}\{1-\exp [-\beta \mathcal{E}(\rho)]\} d \rho,
$$

where the upper integral boundary is determined by $u\left(2 \rho_{u}\right)$ $=k_{B} T[50,51]$. The effective hard-disk radii therefore depend on the geometry of the inclusion, the bending rigidity of the membrane, and on the radius of the vesicle on which the inclusions are placed.

In Fig. 7, the effective hard-disk radii, $r_{\mathrm{hd}}$, are plotted for inclusions with various opening angles as function of the vesicle radius, $R$ (an extremely large radius $R=100 \mu \mathrm{m}$ of the vesicle is used to describe inclusions in planar membranes). The hard-disk radii increase with increasing vesicle radius; the increase in $r_{\text {hd }}$ with $R$ is the stronger, the larger the opening angle $\alpha$ of the inclusion is. For example, the inclusions with $r_{i}=5.5 \mathrm{~nm}$ and $\alpha=0.4 \pi$ can approach each other by diffusion about an order of magnitude closer on a vesicle with radius $R=10 \mathrm{~nm}$ than this is possible on a planar membrane. Consequently, the translational entropy of the inclusions lowers the free energy on the vesicle compared with the planar membrane.

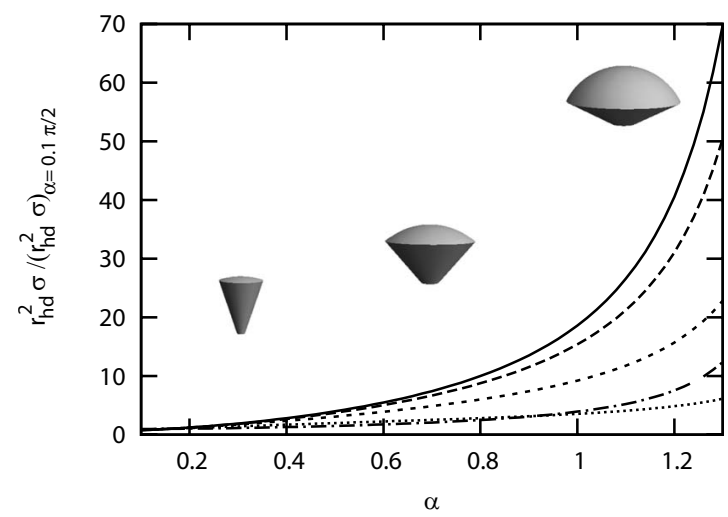

FIG. 8. Area fraction of effective hard disks for inclusions with $r_{i}=5.5 \mathrm{~nm}$ and opening angles $0.1<\alpha<1.3$. For a fixed number of inclusions, cluster formation leads to a larger area fraction of the effective hard disks and finally to crystallization. The area fraction of the effective hard disks with opening angle $\alpha$ is normalized by the area fraction of effective hard disks with $\alpha=0.05 \pi$ : an increase in $\alpha$ corresponds to a decrease in $\sigma$ (compare Fig. 6). The inclusions are placed on vesicles with $\kappa=12 k_{B} T$ and various radii $R=0.1 \mu \mathrm{m}$ (dotted), $R=1 \mu \mathrm{m}$ (short-dashed), $R=10 \mu \mathrm{m}$ (longdashed), and $R=100 \mu \mathrm{m}$ (solid). For comparison, the area fraction of effective hard disks is also shown for $\kappa=1 k_{B} T$ and $R=100 \mu \mathrm{m}$ (dashed-dotted).

As discussed in Sec. II D, cluster formation of inclusions increases their effect on the membrane curvature. The effect of cluster formation on the area fraction of effective hard disks is plotted in Fig. 8. For $\kappa=12 k_{B} T$, which is a typical value for a lipid bilayer, clustering on vesicles with large radii, $R$, strongly increases the area fraction of the effective hard disks. To illustrate the strong effect of the bending rigidity on the effective hard-disk radius, which enters the calculation of the radius via the exponential function in Eq. (12), we plot the effective hard-disk radii for $\kappa=1 k_{B} T$; the increase in the area fraction of the hard disks with $\alpha$ is much smaller than for $\kappa=12 k_{B} T$. Thus, the translational entropy of the inclusions plays a much more important role for smaller $\kappa[52]$.

\section{B. Inclusion entropy and free energy of hard disks}

The free energy of a fluid of hard disks can be very well described by the Carnahan-Starling free energy [53,54]. It is the sum of the ideal-gas free energy, $\mathcal{F}_{\text {id }} / n \approx k_{B} T \log \left(\sigma \Lambda^{2}\right)$, where $\Lambda$ is the thermal wavelength (see, e.g., Ref. [55]) and the excess free energy $\mathcal{F}_{\mathrm{CS}}[56]$. The latter is calculated from the Carnahan-Starling equation of state [54],

$$
\frac{p}{\sigma k_{B} T}=\frac{1}{(1-y)^{2}},
$$

with the hard-disk area fraction, $y=\sigma \pi r_{\text {hd }}^{2}$. Integration of the thermodynamic relation $p=-(\partial F / \partial V)_{T, N}$ finally gives the excess free energy, 


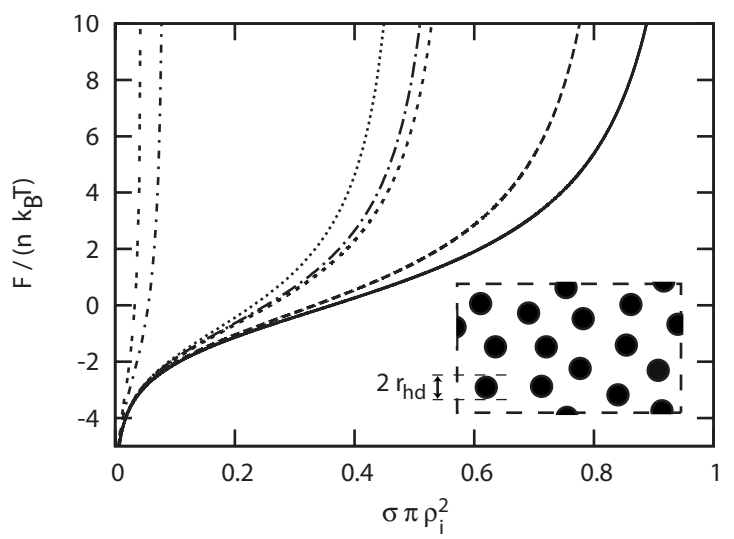

FIG. 9. Carnahan-Starling free energy of hard disks as function of the inclusion area fraction. The system is depicted in the inset with periodic boundary conditions. We plot the free energy for disks with the geometrical projected radii of the inclusions, $r_{\mathrm{hd}}=\rho_{i}$ $=r_{i} \sin \alpha$ (solid line), as well as the free energies for effective hard disks for inclusions with curvature radius $r_{i}=5.5 \mathrm{~nm}$ and various opening angles on vesicles with $\kappa=12 k_{B} T: \alpha=0.16$ and $R=20 \mathrm{~nm}$ (long dashed), $\alpha=0.16$ and $R=100 \mu \mathrm{m}$ (short dashed), $\alpha=0.64$ and $R=20 \mathrm{~nm}$ (long-dashed dotted), $\alpha=0.64$ and $R=100 \mu \mathrm{m}$ (shortdashed dotted), $\alpha=0.82$ and $R=20 \mathrm{~nm}$ (dotted), and $\alpha=0.82$ and $R=100 \mu \mathrm{m}$ (double dashed).

$$
\frac{1}{n} \frac{\mathcal{F}_{\mathrm{CS}}}{k_{B} T}=\int_{0}^{y}\left(\frac{p}{k_{B} T \sigma}-1\right) \frac{d \tilde{y}}{\tilde{y}}=\frac{y}{1-y}-\ln (1-y) .
$$

The Carnahan-Starling excess free energy diverges at the crystallization transition of the effective hard disks.

Because in the fluid as well as in the crystalline phase of the inclusions the squared thermal wavelength enters through the same constant and additive term, we consistently replace it in both cases-without loss of information-by the projected area of the inclusion, $\pi \rho_{i}^{2}$, such that $\widetilde{\mathcal{F}}_{\text {id }} / n$ $\approx k_{B} T \log \left(\sigma \pi \rho_{i}^{2}\right)$.

Usually the translational entropy favors a homogeneous distribution of particles. However, because the effective hard-disk radius depends on the membrane curvature, on a deformable membrane, a homogeneous distribution of inclusion does not need to be the most favorable state. Instead, the inclusion density on the bud can be higher than on the mother vesicle because of the screened repulsive interactions. Figure 9 shows the free energies of a fluid of effective hard disks with curvature radius $r_{i}=5.5 \mathrm{~nm}$ for various opening angles in a lipid bilayer with $\kappa=12 k_{B} T$. For nearly identical curvature radii of vesicle and inclusions, the effective hard-disk radius almost coincides with the geometric hard-disk radius of the inclusion.

\section{Free energy per inclusion in fluid and crystalline phases}

We construct the free energy per inclusion in the crystalline phase from the membrane bending energy and the fluctuation free energy of a harmonic crystal, and in the fluid phase from the sum of the membrane bending energy and the translational entropy of the inclusions [57].

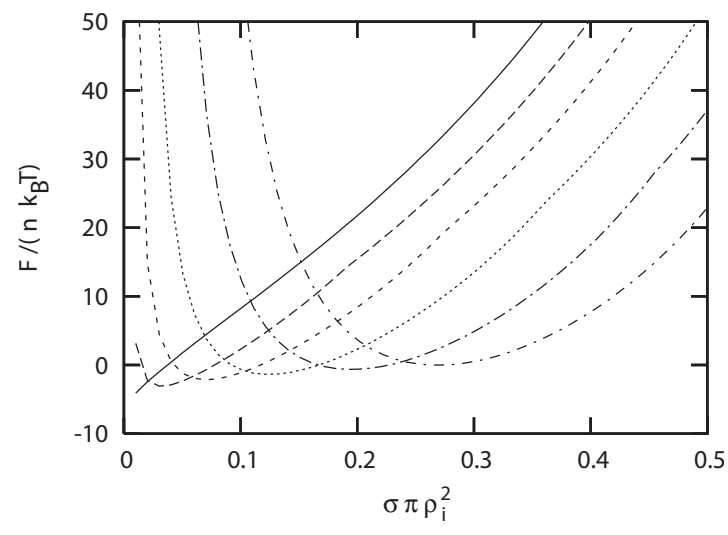

FIG. 10. Free energies as function of the inclusion area fraction, $\sigma \pi \rho_{i}^{2}$, for inclusions with $r_{i}=5.5 \mathrm{~nm}, \alpha=0.82$ in a membrane with $\kappa=12 k_{B} T$ for several vesicle radii: $R=1 \mu \mathrm{m}$ (solid), $R=100 \mathrm{~nm}$ (long-dashed), $R=50 \mathrm{~nm}$ (short-dashed), $R=30 \mathrm{~nm}$ (dotted), $R$ $=20 \mathrm{~nm}$ (long dashed-dotted), $R=15 \mathrm{~nm}$ (short dashed-dotted). For low densities, the inclusions are in a fluid phase and the free energy is given by the membrane bending energy plus the Carnahan-Starling excess free energy for the effective hard disks. For high densities, the inclusions are in a crystalline phase and the free energy is given by the membrane bending energy and the free energy of a harmonic crystal.

For the harmonic crystal, the spring constant $k_{\mathrm{sp}}$ is obtained for a hexagonal lattice with the interaction potential in Eq. (11),

$$
k_{\mathrm{sp}}=\frac{16 \pi \kappa \rho_{i}}{\left(d^{2}-4 \rho_{i}^{2}\right)^{3}}\left(3 d^{2}+4 \rho_{i}^{2}\right) \rho_{i}\left(a^{2}+b^{2}\right)-\left(d^{2}+12 \rho_{i}^{2}\right) a b d .
$$

The free-energy contribution of the positional fluctuations of the inclusions is therefore

$$
\mathcal{F}_{\mathrm{HC}}=k_{B} T \ln \left[\frac{k_{\mathrm{sp}} \Lambda^{2}}{2 \pi}\right]
$$

or, after replacement of the thermal wavelength by the inclusion size,

$$
\tilde{\mathcal{F}}_{\mathrm{HC}}=k_{B} T \ln \left[\frac{k_{\mathrm{sp}} \rho_{i}^{2}}{2}\right] .
$$

Whenever we use the free energy for the crystalline phase in this paper, the Lindemann parameter remains below the critical value for melting of the inclusion crystal [58].

The transition between the fluid and the crystalline phase already occurs below the crystallization transition of the effective hard disks, $\sigma \pi r_{\text {hd }}^{2} \approx 0.7$. In Fig. 10, the free energy per inclusion is plotted for several vesicle radii. Entropy reduces the optimal bud radius for a given inclusion density compared with Eq. (7). However, the bending energy alone still provides a good estimate for the optimal bud radius because of the strong increase in the free energy per inclusion for low inclusion densities (see Fig. 10). 

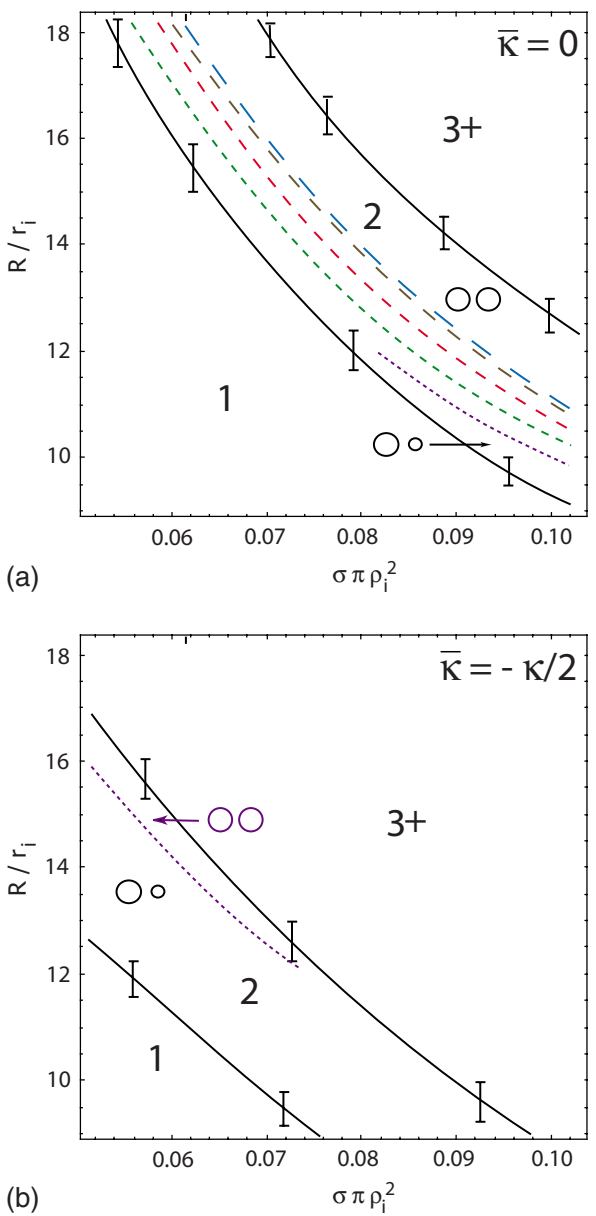

FIG. 11. (Color online) Vesiculation phase diagram for an inclusion density $\sigma$ of inclusions with $r_{i}=5.5 \mathrm{~nm}$ and $\alpha=0.64$ on (initially) a single vesicle of radius $R$. For small $\sigma$ or $R$, the energetically favorable state is the single vesicle; fission, first into two, and finally into three or more vesicles is expected to occur when $\sigma$ and/or $R$ is increased (more than three vesicles are not resolved by the calculation). (a) $\bar{\kappa}=0$ : in the two-vesicle regime, a region where the two vesicles have equal sizes grows with decreasing $\kappa$ and bounds the three-vesicle regime. The lines depict the border between equally and differently sized vesicles for $\kappa=200 k_{B} T$, $\kappa=100 k_{B} T, \kappa=50 k_{B} T, \kappa=30 k_{B} T$, and $\kappa=10 k_{B} T$ (the vesicle sizes are not resolved in the $3+$ region). (b) $\bar{\kappa}=-\kappa / 2$ : fission occurs already for smaller values of $\sigma$ and $R$. In the two-vesicle region the two vesicles have different sizes for $\kappa=200 k_{B} T, \kappa=100 k_{B} T$, $\kappa=50 k_{B} T$, and $\kappa=30 k_{B} T$. For $\kappa=10 k_{B} T$, in a small region both vesicles have equal sizes.

\section{Vesiculation diagrams}

From the total free energy in Sec. III C, we calculate vesiculation diagrams starting with a single vesicle of radius $R$ and a given number of inclusions for several values of $\kappa$. Because the topology changes when buds detach, the value of $\bar{\kappa}$ plays an important role for vesiculation. Figure 11 shows vesiculation diagrams for $\bar{\kappa}=0$ and for $\bar{\kappa}=-\kappa / 2$ (the ratio $\bar{\kappa} / \kappa$ is still under debate; see Ref. [59]). We calculate whether the single vesicle is the most stable state or if fission in two or more smaller vesicles is favorable. With increasing initial vesicle radius and inclusion density, fission becomes more likely to occur-first into two, at even larger $R$ or $\sigma$ into three or more vesicles.

For $\bar{\kappa}=0$, fission in the bending-energy dominated regime produces two smaller vesicles that in general have different sizes; see Eq. (8). If entropy is important, the two vesicles may have equal size. In Fig. 11(a), it is shown that a regime of equally-sized vesicles develops bordering the three-vesicle regime and increasing in size with decreasing $\kappa$. Within the error bars of our calculation, we find that the boundaries between one, two, and three vesicles are independent of the value of the bending rigidity for $10 k_{B} T \leq \kappa \leq 200 k_{B} T$.

For $\bar{\kappa}=-\kappa / 2$, vesiculation takes place for smaller initial vesicle radii and inclusion densities than for $\bar{\kappa}=0$ because each additional vesicle lowers the free energy by $4 \pi \bar{\kappa}$. In the entire two-vesicle regime, both vesicles have different sizes for $\kappa=200 k_{B} T, \kappa=100 k_{B} T, \kappa=50 k_{B} T$, and $\kappa=30 k_{B} T$, while for $\kappa=10 k_{B} T$ a small region of equally sized vesicles is found; see Fig. 11(b).

Note that for bud formation, which has to occur before vesiculation, the value of $\bar{\kappa}$ is irrelevant and the phase diagrams for $\bar{\kappa}=0$ apply (assuming that the buds are connected by catenoidal necks with vanishing bending energy and that the membrane area needed to form the neck is negligible). While the bud is being formed and has not yet detached, the integral over the Gaussian curvature and therefore the contribution of the saddle-splay modulus to the deformation energy stays constant. However, a negative saddle-splay modulus facilitates the neck between two vesicles to break. In this case, the budded state can act as energy barrier for vesiculation that prevents fission, separating a high-energy singlevesicle state and a low-energy state of several smaller vesicles.

\section{BUDDING PATHWAY}

To shed more light on the role of the budding pathway, we compare the typical diffusion time of the inclusions with the relaxation time of the membrane conformation on the same length scale. If the diffusion of inclusions is fast compared with the relaxation time of the membrane, the initial membrane shape is decisive; for a fast membrane relaxation, the initial distribution of inclusions mainly determines the budding process.

The diffusion time is $t_{d}=\lambda^{2} /(4 D)$, where $\lambda$ is a characteristic length scale that the inclusion has diffused and $D$ is the diffusion coefficient of the inclusion. A typical value is $D$ $=1 \mu \mathrm{m}^{2} \mathrm{~s}^{-1}$ for the diffusion of lipids and the diffusion coefficient for proteins in cell membranes can be up to two orders of magnitude smaller [60]. The relaxation time of the membrane is $t_{r}=\eta \lambda^{3} /\left(2 \pi^{3} \kappa\right)$ [61], where $\eta=1 \mathrm{mPa} \approx 2.4$ $\times 10^{-10} k_{B} T \mathrm{~s} \mathrm{~nm}^{-3}$ is the viscosity of the surrounding water and $\lambda$ is the wavelength of the membrane undulations. From the cubic versus the quadratic dependence on $\lambda$, we find that for small $\lambda, t_{r}<t_{d}$, while for large $\lambda, t_{r}>t_{d}$.

For $\kappa=10 k_{B} T$ and $D=1 \mu \mathrm{m}^{2} \mathrm{~s}^{-1}$ (which is an upper bound for the diffusion coefficient of proteins), we find that $t_{r}=t_{d}$ for $\lambda \approx 0.6 \mathrm{~mm}$. This length is much larger than $10 \mu \mathrm{m}$, which is the size of cells [62] or giant unilamellar vesicles [63], thus the initial aggregation of inclusions is dif- 
fusion limited. We therefore assume that inhomogeneities in the protein distribution on the membrane will immediately lead to a membrane deformation that minimizes the bending energy. The larger the initial inclusion density, the larger the spontaneous curvature of the membrane [compare Eq. (7)] and the smaller the size of the buds that are formed.

Bud formation is initialized in some regions of the membrane that have a noticeably higher inclusion density than others. The relative protein density fluctuations decrease with the size of a membrane patch which is considered. If we assume a random distribution of inclusions, for a patch with $N$ inclusions the relative fluctuations of the inclusion number are of the order of $N^{-1 / 2}$. Thus, for small patches, the inhomogeneities are strongest and budding will preferably occur on the smallest possible length scale. A small average membrane curvature with appropriate sign further attracts proteins to those regions where the bending energy per inclusion is already reduced. However, this clustering of inclusions during the budding process is hindered by a ring with opposite membrane curvature that forms the neck of a growing bud. This ring acts as energetic barrier that prevents further inclusions to enter a patch of the membrane where budding has already started [64]. Because of the neck formation and the diffusion-limited budding process, the bud size is roughly determined by the initial inclusion density on the membrane.

\section{COMPARISON WITH SIMULATION RESULTS}

Budding due to membrane inclusions has been studied recently with computer simulations [36,37]. In Ref. [37], entire vesicles with inclusions are simulated where the membrane is modeled as a dynamically triangulated surface. In Ref. [36], coarse-grained model lipids are used to study budding for planar bilayer patches.

In Fig. 12, the different contributions to the free energy per inclusion needed to graft ten inclusions with given projected radius $\rho_{i}=2.5 \mathrm{~nm}$ on a vesicle with radius $R$ $=15 \mathrm{~nm}$ are plotted as function of the opening angle $\alpha$. The inclusions are in the fluid phase. For comparison, we also plot the simulation data of Ref. [37], shifted by $\Delta F$ $=-10.5 k_{B} T$ because our model does not account for thermal undulations of the membrane conformation. This energy difference is extracted from the simulation data for $T=300 \mathrm{~K}$ and for $T=3 \mathrm{~K}$; see Fig. 6A in Ref. [37]. However, the very good match is somewhat fortuitous because we replace the thermal wavelength in the ideal gas free energy by the inclusion size, such that it is similar to the free energy obtained on a triangulated vesicle with a bond length that approximately equals to the inclusion diameter; compare [65].

We consider curvature radii of the inclusions that are both smaller and larger than the curvature radius of the vesicle. For $\alpha \rightarrow 0$ and $r_{i} \rightarrow \infty$, such that $\rho_{i}=2.5 \mathrm{~nm}$, bending energy is needed to insert the flat inclusion into the curved vesicle. This bending energy cost decreases with increased opening angle $\alpha$ and is zero for $\alpha \approx 0.17$ where the curvature radius of the inclusion equals the curvature radius of the vesicle. If $\alpha$ is further increased, the bending energy per inclusion continues to decrease as more and more of the vesicle area consists of catenoidal patches around the inclusions. For

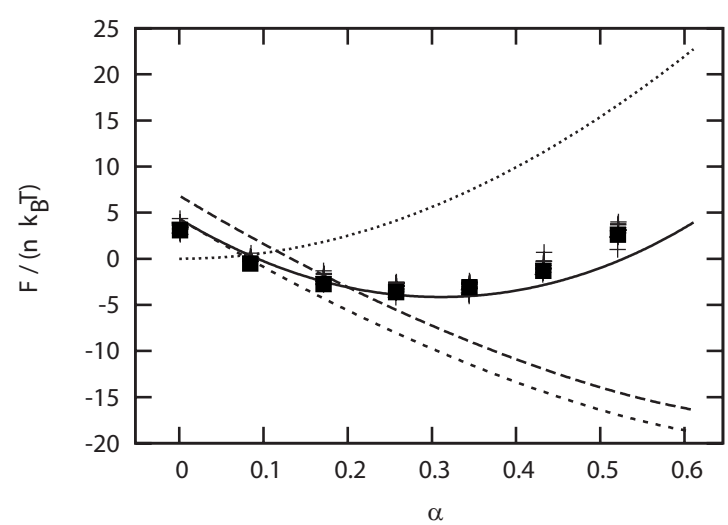

FIG. 12. Energies per inclusion needed to place ten inclusions of size $\rho_{i}=2.5 \mathrm{~nm}$ on a vesicle with $R=15 \mathrm{~nm}, \kappa=20 k_{B} T$, and $\bar{\kappa}$ $=-20 k_{B} T$, as function of $\alpha$. Lines show bending energy (longdashed), bending energy and inclusion entropy (short-dashed), saddle-splay energy (dotted), and total energy (solid). For comparison, we also plot the simulation data of Ref. [37] (symbols indicate different calculation methods [37]) shifted by $\Delta F=-10.5 k_{B} T$ (see main text). The deviation of the simulations and our theory for $\alpha$ $\gtrsim 0.4$ might be due to the surface tension used in the simulation, which is not included in our model.

$\alpha \approx 1.18$, i.e., for even larger opening angles than plotted in the figure, the inclusions have optimal density and the bending energy gained by grafting all ten inclusions to the vesicle is $8 \pi \kappa$.

In addition to the bending energy, there is an energy cost $\mathcal{E}_{\bar{\kappa}}$ for grafting that arises due to the saddle-splay modulus, which has been chosen to be $\bar{\kappa}=-\kappa=-20 k_{B} T$ for consistency with Ref. [37]. The energy cost per inclusion only depends on the geodesic curvature of the membrane at the inclusion, i.e., on the opening angle $\alpha$, which implies $\mathcal{E}_{\bar{\kappa}}=-2 \pi \bar{\kappa}(1$ $-\cos \alpha$ ). Therefore, it is independent of vesicle radius and inclusion density and is only important to calculate the chemical potential for the inclusions on the surface; the budding transition at given inclusion density in the membrane is independent of $\bar{\kappa}$.

In the simulations presented in Ref. [36], budding is induced by inclusions with $r_{i}=5.5 \mathrm{~nm}$ that are initially placed in a regular array on a planar membrane with $\kappa=12 k_{B} T$. Under the assumption that the initial inclusion density, $\sigma$ $=2 \times 10^{-3} \mathrm{~nm}^{-2}$, determines the bud size (see Sec. IV), we can roughly predict the bud radius from Eq. (7). Possible bud radii are estimated by comparing the free energies for different vesicle radii in Fig. 10 at the initial inclusion density.

The parameters for which the free energies are plotted in Fig. 10 are chosen to match the bending rigidity and the inclusion geometry of the "large inclusions" in Ref. [36] with $\alpha=0.26 \pi$ [66]. For an initial inclusion density in the planar membrane, $\sigma=0.002 \mathrm{~nm}^{-2} \approx 0.15 \sigma \pi \rho_{i}^{2}$, which is estimated by visual inspection from the simulation snapshots, we find that the inclusions are in the crystalline phase on the vesicle with $R=1 \mu \mathrm{m}$ (i.e., in a planar membrane). The free energy per inclusion is about $10 k_{B} T$. Significantly smaller free energies per inclusion can be found for vesicle radii $22 \mathrm{~nm} \leqslant R$ $\lesssim 100 \mathrm{~nm}$; the optimal vesicle radii are $30 \mathrm{~nm} \lesssim R$ $\lesssim 60 \mathrm{~nm}$ with free energies per inclusion of about $-1 k_{B} T$. 
These radii agree well with the observed radius $R=30 \mathrm{~nm}$ of the bud that forms in the simulations as final state via an initially slightly larger bud. The optimal bud radius from Eq. (7) is approximately $37 \mathrm{~nm}$.

Similarly, for the "very large inclusions" in Ref. [36] ( $\alpha$ $=0.39 \pi$ ) we predict bud radii, $15 \mathrm{~nm} \lesssim R \lesssim 20 \mathrm{~nm}$, as observed in the simulations; based on bending energy only we find from Eq. (7) $R \approx 11 \mathrm{~nm}$. For the "small inclusions" in Ref. [36] $(\alpha=0.20 \pi)$ that are already in the planar membrane almost in the fluid phase, our model predicts for the 36 inclusions studied in the simulations a maximal gain for the free energy per inclusion of $\approx 1.5 k_{B} T$ for $R=38 \mathrm{~nm}$ and a strong decrease in this energy gain for smaller vesicle radii. Already for $R=34 \mathrm{~nm}$ and 29 inclusions, the energy per inclusion on the bud and in the plane are approximately equal. Larger energy gains are possible for larger bud radii, for which many more than the simulated 36 inclusions and a larger area of the bilayer patch are needed. From these considerations, it is not surprising that no budding is observed for the small inclusions in the simulations of Ref. [36].

\section{CONCLUSIONS}

We have calculated the membrane-mediated interaction of conical inclusions in a lipid bilayer and the inclusion entropy, which allow the prediction of budding transitions and vesiculation. Our model is based on the membrane bending energy; with this contribution alone, the spontaneous curvature of a bilayer with inclusions as well as budding can be predicted for many systems, ranging from protein inclusions to viral budding. Although the interaction between the inclusions by membrane deformation is repulsive, the screening of the repulsive interaction due to the average membrane curvature allows higher inclusion densities on a bud than in the initial vesicle. Translational entropy of the inclusions favors the formation of equally sized daughter vesicles and lifts the degeneracy that is found for states with vanishing bending energy.

From our calculations, the following picture of the effect of the bilayer deformation by curved inclusions emerges. For low inclusion density, the membrane around each inclusion assumes a catenoid shape of vanishing curvature energy. At optimal inclusion density, the catenoids are closely packed and the bending energy for the entire vesicle vanishes. For high inclusion density, the boundary conditions for the membrane deformation around the inclusion do not allow the formation of catenoidal patches and the inclusions always feel the membrane-mediated repulsive interaction with neighboring inclusions. In this regime, bud formation can occur.

If we assume a specific biological mechanism that leads to formation of clusters with well-defined and limited size, we find that such a mechanism can induce bud formation and vesiculation without the need to insert additional conical proteins into the cell membrane: cluster formation reduces the preferred curvature radius of the membrane. We quantify the effect of aggregation by the coagulation factor, which describes how the preferred curvature radius for a fixed amount of inclusions changes with the cluster size.

In general, our analytical model is applicable for a wide range of length scales and inclusion geometries. Computer simulations are usually designed only for a specific length scale, e.g., a length scale comparable to the length scale of lipids in Ref. [36] or the length scale of entire vesicles in Ref. [37]. The good agreement with the simulation results of Refs. [36,37] suggests that the approximations used in our calculations are justified.

We argue that the undulation-induced attraction can be neglected compared with the deformation-induced repulsion and the translational entropy of the inclusions for $\kappa \alpha^{2}$ $\gtrsim 15 k_{B} T /(2 \pi)$, i.e., $\alpha \gtrsim 0.35$ for $\kappa=20 k_{B} T$. For example, the BAR (Bin/amphiphysin/Rvs) domains induce a local membrane curvature with an opening angle $\alpha \approx 0.4$ [4]. Clathrin can induce a variety of opening angles $[67,68]$.

The number of the inclusions per bud is determined by the budding process. Around a growing bud, a neck forms that presents an energetic barrier for the diffusion of inclusions. Because the deformation of the lipid membrane typically occurs much faster than the diffusion of the inclusions within the membrane, the number of inclusions per bud is well determined by the initial inclusion density in the membrane. From this, we can estimate a range of possible bud radii, which agrees well with the simulations in Ref. [36].

It would be interesting to test the validity of our model in the limits of (a) very small inclusions, such as lipids with large headgroups, when the description of the lipid membrane by curvature elastic constants may not be appropriate and (b) very floppy membranes, when neglecting the thermal membrane undulations may not be justified.

\section{ACKNOWLEDGMENTS}

We acknowledge helpful discussions with G. Nägele, R. G. Winkler, J. L. McWhirter, K. Mecke, R. Golestanian, M. Deserno, and M. Oettel.
[1] A. D. Dupuy and D. M. Engelman, Proc. Natl. Acad. Sci. U.S.A. 105, 2848 (2008).

[2] P. Sens, L. Johannes, and P. Bassereau, Curr. Opin. Cell Biol. 20, 476 (2008).

[3] G. Drin, J. F. Casella, R. Gautier, T. Boehmer, T. U. Schwartz, and B. Antonny, Nat. Struct. Mol. Biol. 14, 138 (2007).

[4] P. D. Blood and G. A. Voth, Proc. Natl. Acad. Sci. U.S.A. 103, 15068 (2006).

[5] T. Auth and G. Gompper, Phys. Rev. E 72, 031904 (2005).
[6] T. Bickel and C. M. Marques, Eur. Phys. J. E 9, 349 (2002).

[7] M. G. J. Ford, I. G. Mills, B. J. Peter, Y. Vallis, G. J. K. Praefcke, P. R. Evans, and H. T. McMahon, Nature (London) 419, 361 (2002).

[8] J. L. Harden and F. C. Mackintosh, Europhys. Lett. 28, 495 (1994).

[9] H. Hagerstrand, L. Mrowczynska, U. Salzer, R. Prohaska, K. A. Michelsen, V. Kralj-Iglic, and A. Iglic, Mol. Membr. Biol. 23, 277 (2006). 
[10] M. M. Kozlov, Nature (London) 447, 387 (2007).

[11] B. Antonny, Curr. Opin. Cell Biol. 18, 386 (2006).

[12] R. Shlomovitz and N. S. Gov, Phys. Rev. Lett. 98, 168103 (2007).

[13] W. Römer et al., Nature (London) 450, 670 (2007).

[14] H. T. McMahon and J. L. Gallop, Nature (London) 438, 590 (2005).

[15] H. J. Gao, W. D. Shi, and L. B. Freund, Proc. Natl. Acad. Sci. U.S.A. 102, 9469 (2005).

[16] W. T. Góźdź, Langmuir 23, 5665 (2007).

[17] T. Auth and G. Gompper, Phys. Rev. E 68, 051801 (2003).

[18] I. Tsafrir, Y. Caspi, M. A. Guedeau-Boudeville, T. Arzi, and J. Stavans, Phys. Rev. Lett. 91, 138102 (2003).

[19] F. Campelo, J. M. Allain, and M. Ben Amar, Europhys. Lett. 77, 38006 (2007).

[20] T. Baumgart, S. T. Hess, and W. W. Webb, Nature (London) 425, 821 (2003).

[21] C. K. Haluska, W. T. Gozdz, H. G. Döbereiner, S. Forster, and G. Gompper, Phys. Rev. Lett. 89, 238302 (2002).

[22] W. T. Góźdź and G. Gompper, Europhys. Lett. 55, 587 (2001).

[23] R. Bruinsma and P. Pincus, Curr. Opin. Solid State Mater. Sci. 1, 401 (1996).

[24] M. Goulian, Curr. Opin. Colloid Interface Sci. 1, 358 (1996).

[25] M. Goulian, R. Bruinsma, and P. Pincus, Europhys. Lett. 22, 145 (1993).

[26] M. Goulian, R. Bruinsma, and P. Pincus, Europhys. Lett. 23, 155 (1993)

[27] T. R. Weikl, M. M. Kozlov, and W. Helfrich, Phys. Rev. E 57, 6988 (1998).

[28] T. Chou, K. S. Kim, and G. Oster, Biophys. J. 80, 1075 (2001).

[29] K. S. Kim, J. Neu, and G. Oster, Biophys. J. 75, 2274 (1998).

[30] P. G. Dommersnes, J. B. Fournier, and P. Galatola, Europhys. Lett. 42, 233 (1998).

[31] K. S. Kim, J. C. Neu, and G. F. Oster, Europhys. Lett. 48, 99 (1999).

[32] W. Helfrich and T. R. Weikl, Eur. Phys. J. E 5, 423 (2001).

[33] T. R. Weikl, Europhys. Lett. 54, 547 (2001).

[34] R. Golestanian, M. Goulian, and M. Kardar, Phys. Rev. E 54, 6725 (1996).

[35] R. Golestanian, M. Goulian, and M. Kardar, Europhys. Lett. 33, 241 (1996).

[36] B. J. Reynwar, G. Illya, V. A. Harmandaris, M. M. Müller, K. Kremer, and M. Deserno, Nature (London) 447, 461 (2007).

[37] E. Atilgan and S. X. Sun, J. Chem. Phys. 121, 10392 (2004).

[38] In general, the boundary conditions are given by $h\left(\rho_{b}\right)$ $=h_{0}\left(\rho_{b}\right)$ and $h^{\prime}\left(\rho_{b}\right)=h_{1}\left(\rho_{b}\right)$ for the inner and the outer boundaries each or alternatively-if the values of $h$ and $h^{\prime}$ are not known at the boundary-by the natural boundary conditions $\left.\partial_{\rho} \Delta h(\rho)\right|_{\rho=\rho_{b}}=0$ and $\left.\Delta h(\rho)\right|_{\rho=\rho_{b}}=0$, respectively [69].

[39] Part of a small sphere (the inclusion) is matched to the narrow part of the catenoid such that the slope is continuous, which again is matched to a larger sphere (the vesicle); see Fig. 3.

[40] The equations are applicable for large $n$, but also in the exact relations the inclusion geometry enters only as a single variable, $|a| \rho_{i}$.

[41] Budding in this regime of inclusion concentration might be possible for a negative value of $\bar{\kappa}$.

[42] Equation (7) is valid for large $n$; for very small vesicles the approximation breaks down and in addition discretization effects become important.

[43] J. J. Sieber, K. I. Willing, C. Kutzner, C. Gerding-Reimers, B Harke, G. Donnert, B. Rammner, C. Eggeling, S. W. Hell, H. Grubmueller, and T. Lang, Science 317, 1072 (2007).

[44] We neglect the contribution of the geodesic curvature, the influence of which is characterized by $\bar{\kappa}$.

[45] In the original method that applies to steep potentials, $\mathcal{E}\left(\rho_{u}\right)$ $=0$ is used.

[46] R. R. Netz and P. Pincus, Phys. Rev. E 52, 4114 (1995).

[47] J. B. Fournier and P. G. Dommersnes, Europhys. Lett. 39, 681 (1997).

[48] T. R. Weikl, Phys. Rev. E 66, 061915 (2002).

[49] J. A. Barker and D. Henderson, J. Chem. Phys. 47, 2856 (1967).

[50] D. Henderson and J. A. Barker, Phys. Rev. A 1, 1266 (1970).

[51] R. O. Watts and D. Henderson, J. Chem. Phys. 50, 1651 (1969).

[52] It is not clear whether for $\kappa \simeq 1 k_{B} T$ the thermal undulations of the membrane can still be neglected, i.e., whether our model applies.

[53] N. F. Carnahan and K. E. Starling, J. Chem. Phys. 51, 635 (1969).

[54] M. J. Maeso and J. R. Solana, J. Chem. Phys. 99, 548 (1993).

[55] C. Gögelein, G. Nägele, R. Tuinier, T. Gibaud, A. Stradner, and P. Schurtenberger, J. Chem. Phys. 129, 085102 (2008).

[56] J.-P. Hansen and I. R. McDonald, Theory of Simple Liquids, 3rd ed. (Academic Press, London, 2006).

[57] The cell approximation [70] for the free energy of the crystalline phase is not useful, because the effective hard disks overlap already at densities that are slightly higher than the crystallization transition of the inclusions and below densities that are observed in simulations.

[58] C. Eisenmann, P. Keim, U. Gasser, and G. Maret, J. Phys.: Condens. Matter 16, S4095 (2004).

[59] M. Deserno, Macromol. Rapid Commun. 30, 752 (2009).

[60] Y. I. Henis, in Biomembranes: Physical Aspects, edited by M. Shinitzky (VCH, Weinheim, 1993), pp. 279-339.

[61] N. Gov, A. G. Zilman, and S. Safran, Phys. Rev. Lett. 90, 228101 (2003).

[62] B. Alberts, D. Bray, and J. Lewis, Molecular Biology of the Cell, 4th ed. (Garland Science, New York, 2002).

[63] J. Pecreaux, H. G. Döbereiner, J. Prost, J. F. Joanny, and P. Bassereau, Eur. Phys. J. E 13, 277 (2004).

[64] By this mechanism, the final number of inclusions per bud is already determined in an early stage of bud formation. The area of lipid bilayer per bud can adjust during the budding process and is regulated by the number of inclusions on the bud.

[65] A.-S. Smith and U. Seifert, Phys. Rev. E 71, 061902 (2005).

[66] Many numerical values of the parameters in the figures have been chosen such that they correspond to those in Ref. [36].

[67] T. Kohyama, D. M. Kroll, and G. Gompper, Phys. Rev. E 68, 061905 (2003).

[68] J. Heuser and L. Evans, J. Cell Biol. 84, 560 (1980).

[69] N. Dan, A. Berman, P. Pincus, and S. A. Safran, J. Phys. II 4, 1713 (1994).

[70] G. Döge, K. Mecke, J. Moller, D. Stoyan, and R. P. Waagepetersen, Int. J. Mod. Phys. C 15, 129 (2004). 